\title{
Estrogen induces angiogenesis of the female rabbit bladder
}

\author{
Alpha Dian-Yu Lin ${ }^{1,2,4}$, Anita Mannikarottu ${ }^{1,2}$, Barry A Kogan ${ }^{2}$, Catherine Whitbeck ${ }^{1,3}$, Paul Chichester ${ }^{1,3}$, \\ Robert E Leggett ${ }^{1,3}$ and Robert M Levin ${ }^{1,2,3}$ \\ ${ }^{1}$ Albany College of Pharmacy, ${ }^{2}$ Albany Medical College, ${ }^{3}$ Stratton VA Medical Center, 106 New Scotland Ave, Albany, New York 12208, USA \\ ${ }^{4}$ Taichung Poah-Ai Hospital, Taipei, Taiwan \\ (Requests for offprints should be addressed to R M Levin; Email: levinr@acp.edu)
}

\begin{abstract}
Postmenopausal bladder dysfunction has been speculated to involve decreased circulating estrogen levels. It is our hypothesis that estrogen induces bladder dysfunctions by modulating blood flow to the bladder, i.e. low estrogen reduces blood flow to the bladder, whereas high estrogen increases blood flow. Our previous studies have demonstrated that estrogen administration in female rabbits induces a 'functional hypertrophy' of the urinary bladder smooth muscle represented by increased smooth muscle mass, which corresponds to increased contractile responses to all forms of stimulation. The present study investigates the effect of estrogen on vasculature density and distribution. Twentyfour female New Zealand white rabbits were separated into six groups of four rabbits each. Group 1 served as controls. Groups 2-6 were ovariectomized. Two weeks after ovariectomy (Ovx), groups 3-6 were given 17- $\beta$ estradiol
\end{abstract}

( $1 \mathrm{mg} / \mathrm{kg}$ per day) by s.c. implant for $1,3,7$, and 14 days respectively. Blood vessel density and distribution were evaluated by immunohistochemistry and quantitative image analyses. Ovx resulted in significant vascular degeneration and decreased density, whereas estradiol administration mediated a significant angiogenic effect characterized by increased vascular density, and distribution of new vasculature within the smooth muscle bundles of the detrusor. Estradiol-induced vasculogenesis corresponds with our previously demonstrated increase in blood flow to the bladder and increased contractility. The most interesting aspect of these studies is the increased vascularization localized within the muscle bundles rather than between the muscle bundles, which may be important in the link between estrogen and increased incidence of cancers.

Journal of Endocrinology (2006) 190, 241-246

\section{Introduction}

Postmenopausal urinary bladder dysfunctions including recurrent urinary tract infection and incontinence may be associated with decreased serum estrogen ( $\mathrm{Hu}$ et al. 2004, Tinelli et al. 2005). One mechanism that has been speculated to be involved in these bladder dysfunctions involves estrogen-mediated blood flow changes to the bladder (Parekh et al. 2004). In our previous studies, Ovx resulted in decreased blood flow to the bladder mucosa and muscle compartments resulting in mucosal and smooth muscle atrophy, increased collagen synthesis and distribution, and decreased contractility. Estrogen supplementation reversed the effects of Ovx and resulted in smooth muscle hypertrophy and mucosal hyperplasia, and enhanced contractility (Aikawa et al. 2003).

Angiogenesis, the formation of new blood vessels, is a complex process involving an increased expression of cell matrix degrading proteases, enhanced endothelial cell proliferation and migration, and vessel formation. Estrogen is proposed to modulate angiogenesis directly via effects on endothelial cells
(ECs; Losordo \& Isner 2001). The positive correlation between estrogen receptor expression, angiogenic activity and breast tumor invasiveness also supports an angiogenic effect of estrogen (Weidner et al. 1991, Vartanian et al. 1994).

In our earlier study, estradiol administration resulted in a functional hypertrophy that is manifested by an increased volume-fraction of smooth muscle within the detrusor, which is accompanied by a proportional increase in contractility. It is our hypothesis that the functional hypertrophy is accompanied by angiogenesis such that the hypertrophied bladder is vascularized simultaneously with the hypertrophy. In addition, we investigated the expression of two highly potent angiogenic markers, vascular endothelial growth factor (VEGF) and hypoxia inducible factor (HIF-1 $\alpha$ ) in the hypertrophied bladder.

\section{Materials and Methods}

\section{Animals}

Twenty-four female New Zealand White female rabbits were separated into six groups of four rabbits each. Group 1 served 
as controls. Groups 2-6 were ovariectomized. Two weeks after ovariectomy (Ovx), groups 3-6 were given 17- $\beta$ estradiol (1 mg/kg per day) for $1,3,7$, and 14 days respectively. Estradiol was administered via surgical implantation of a slow-release pellet in the subscapular region under ketamine/ xylazine $(25 \mathrm{mg} / \mathrm{kg}) /(10 \mathrm{mg} / \mathrm{kg})$ anesthesia.

At the end of the appropriate time periods, each rabbit was anesthetized with pentobarbital, the bladder excised through a mid-line incision, and the rabbits euthanized.

\section{Ovariectomy}

Each rabbit was anesthetized with isoflorane (1-3\%). Both ovaries were excised through bilateral incisions that were then closed with 2-0 silk.

\section{Histology}

Each full thickness strip of tissue was fixed in 10\% neutral buffered formalin for 6-8 h and embedded in paraffin blocks. The tissue blocks were sectioned at $5 \mu \mathrm{m}$ and were used for the immunostaining studies.

\section{Immunostaining for vascular endothelium using CD31}

Tissue sections were stained with the mouse monoclonal antibody anti-CD31, clone JC/70A (Dako, Carpinteria, CA, USA) on the Ventana ES automated immunostainer (Ventana Medical Systems, Tucson, AZ, USA) using an avidin-biotin and diaminobenzidine (DAB) chromagin detection kit followed by a light hematoxylin counterstain. The detection kit from Ventana was used with the dilutions, times, and temperatures set by the supplier, except for the following. The antigen retrieval was done with Protease 1 (Ventana) for 4 min. Initial blocking was done with serum-free protein block (Dako) for $12 \mathrm{~min}$ and $100 \mu \mathrm{l}$ of CD31 primary antibody was applied for $32 \mathrm{~min}$ at a dilution of 1:20 in antibody diluent (Dako). The secondary antibody in the kit is substituted with biotinylated goat anti-mouse secondary predilute (Lab Vision, Fremont, CA, USA). After immunostaining, all the slides were dehydrated through graded alcohols and two changes of xylene and then mounted with Permount (Fisher Scientific, Pittsburgh, PA, USA) mounting medium. Quantification of blood vessel density and circumference was obtained from the immunostained transverse sections by the image system (Image Pro Plus, Media Cybernetics, Silver Springs, MD, USA). A minimum of 12 sections and four fields per section per bladder detrusor were evaluated. All vessels within the field were counted and then calculated as the mean number of vessels per unit area.

\section{Western blotting}

VEGF and HIF- $1 \alpha$ were utilized as indicators of angiogenesis. Frozen bladder tissues $(100 \mathrm{mg})$ were pulverized, while immersed in liquid nitrogen using a mortar and was homogenized in buffer containing $20 \%$ glycerol, $50 \mathrm{mM}$ Tris- $\mathrm{HCl}(\mathrm{pH} 6 \cdot 8), 0.5 \%$ (v/v) Tween-20, and protease inhibitors $(0.5 \mathrm{mM}$ phenylmethanesulfonyl fluoride, $2 \mu \mathrm{M}$ pepstatin, $2 \mu \mathrm{M}$ anti-pain and $0.1 \mathrm{mg} / \mathrm{ml}$ trypsin inhibitor). The homogenate was centrifuged $(38000 \mathrm{~g})$ for $15 \mathrm{~min}$ using a Sorvall centrifuge and the supernatant was collected. After gently mixing the samples in SDS (final concentration, 1\%), they were boiled for $4 \mathrm{~min}$ and centrifuged at 10000 r.p.m. for $15 \mathrm{~min}$. The supernatant was collected and the protein concentration was determined using the protein assay kit (Bio-Rad). Equal amounts $(20 \mu \mathrm{g})$ of total protein from each group were loaded on 12\% SDS-PAGE gels and transferred to Immobilon-P membranes with Towbin buffer (25 mM Tris, $192 \mathrm{mM}$ Gly, and 20\% (v/v) methanol). The membranes were blocked with $5 \%$ nonfat milk in $0.05 \%$ Tween-20 in PBS for $1 \mathrm{~h}$ at $37^{\circ} \mathrm{C}$. They were then incubated with primary antibodies, VEGF (1:1000) and HIF-1 $\alpha$ (1:500) (Novus Biologicals, Inc., Littleton, CO, USA) respectively. The membranes were washed with washing buffer $(20 \mathrm{mM}$ Tris, $500 \mathrm{mM} \mathrm{NaCl}$, and 0.05\% Tween-20) and incubated with secondary antibody (goat anti-mouse $\operatorname{IgG}$ at $1: 10$ 000). The substrates were visualized by echochemiluminescence and analyzed by the image system (Kodak).

\section{Statistical analysis}

All the data are expressed as the mean \pm s.E.M. with $P<0 \cdot 05$ considered statistically significant. The $t$-test was applied using Sigma Stat, version 2.03 (SPSS, Chicago, IL, USA).

These studies were approved by the Institutional Committee on the Care and Use of Animals of the Stratton Veterans Affairs Medical Center.

\section{Results}

Figure 1 shows the bladder weights. Bladder weight progressively increased reaching a maximum at 7 days and remaining at that level for 14 days.

Figure 2 shows representative immunohistochemical sections of bladder detrusor stained for vascular endothelium with CD-31. Estrogen not only mediated an obvious increase in blood vessel density, it also resulted in a significant change in the distribution of the vessels. As observed in both the control and Ovx bladders, the large majority of blood vessels travel between the discrete muscle bundles and not through and between the muscle cells. However, following estrogen supplementation, the new blood vessels are observed both within the muscle bundles and between the individual muscles cells (Fig. 2C), ensuring the bladder of adequate oxygen delivery to the hypertrophied muscle bundles.

Figure 3 shows the mean density of the vessels per group. Blood vessel density significantly decreased 2 weeks after ovariectomy, which corresponds with the decreased blood flow demonstrated in earlier studies. Blood vessel density 


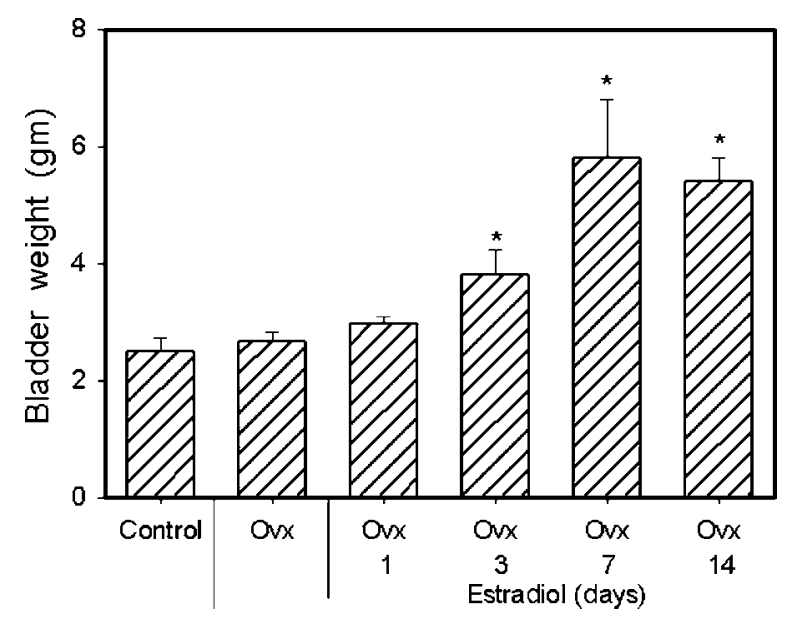

Figure 1 Changes in bladder weight 2 weeks after ovariectomy and estrogen treatments. There is an increase in bladder weight 7 days after ovariectomy and estrogen treatment, which remain high throughout the rest of the experimental period. Each bar is the mean \pm s.E.M. of four individual rabbits. *Significantly different from both control and Ovx, $P<0 \cdot 05$.

increased significantly over the 2 -week estrogen supplementation period. Figure 4 shows the mean circumference of the blood vessels within the bladder as a percentage of all blood vessels in that group. It is easily seen that estrogen supplementation mediated a significant shift to smaller size vessels (microvessels), which is characteristic of angiogenesis.
Figure 5 demonstrates that VEGF content was enhanced by Ovx, and then decreased toward control values at 1-day estradiol administration. VEGF rose to levels higher than both control and Ovx at 7 and 14 days of estradiol administration.

Figure 6 demonstrates that HIF- $1 \alpha$ content follows the same time course as was seen with VEGF. HIF- $1 \alpha$ is significantly increased in the Ovx group, decreases to below control levels at 1-day estrogen and then progressively rises to high levels in the 7 and 14 days estrogen groups. These patterns for VEGF and HIF $1 \alpha$ are consistent with the angiogenic effect of estrogen observed in the 7- and 14-day estrogen groups.

\section{Discussion}

Hypoxia is a well-known stimulant for angiogenesis. However, even though the expressions of VEGF and HIF$1 \alpha$ are increased, vascular density decreases because of the extent of hypoxic damage to the vascular endothelium including stimulating apoptosis of the endothelium (Shweiki et al. 1992, Vincent et al. 2000). A recent report by Kazi et al. (2005) shows that HIF-1 is an important mediator of estrogen-induced VEGF expression in the uterus.

Angiogenesis is the process of generating new capillary blood vessels, which includes at least four distinct properties
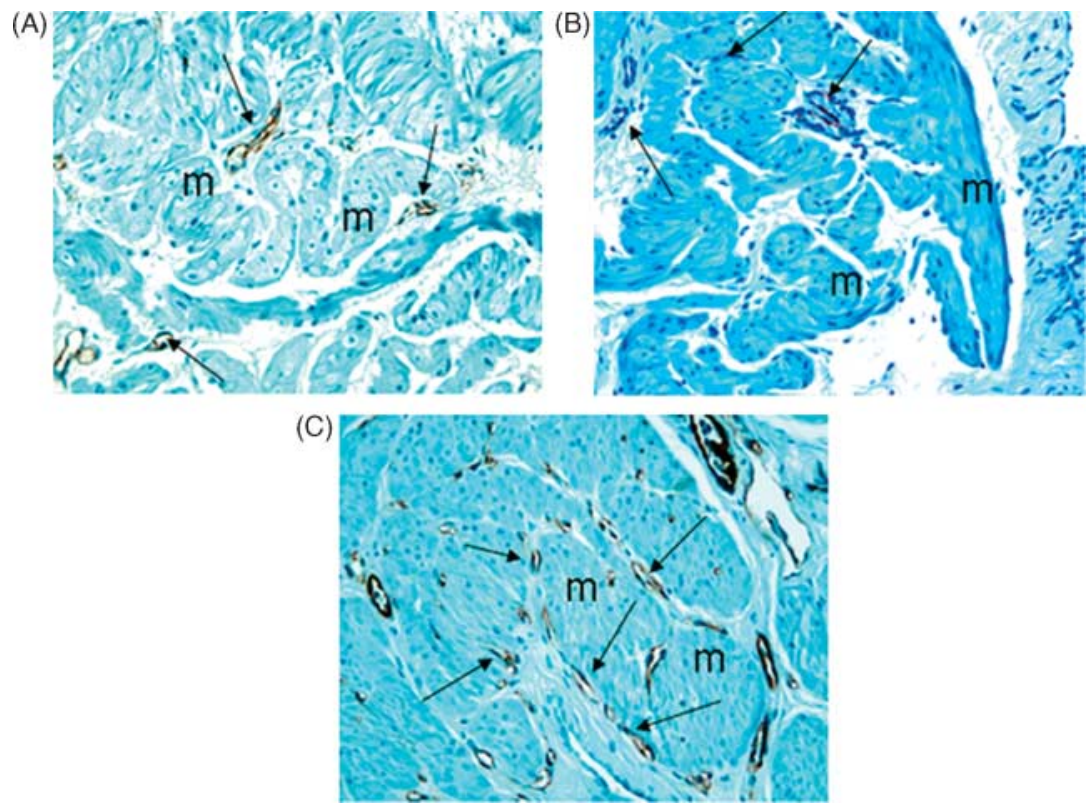

Figure 2 Representative photo-micrographs of control, Ovx, and Ovx followed by 2 weeks of estrogen, showing vascularity using CD-31 immunohistochemistry. ( $A$ and $B$ ) In both the control and Ovx bladders, the large majority of blood vessels travel between the discrete muscle bundles (black arrows). (C) Following estrogen supplementation, the new blood vessels are observed both within the muscle bundles and between the individual muscles cells (black arrows). Detrusor smooth muscles are marked as m. All figures in $200 \times$ magnification. 


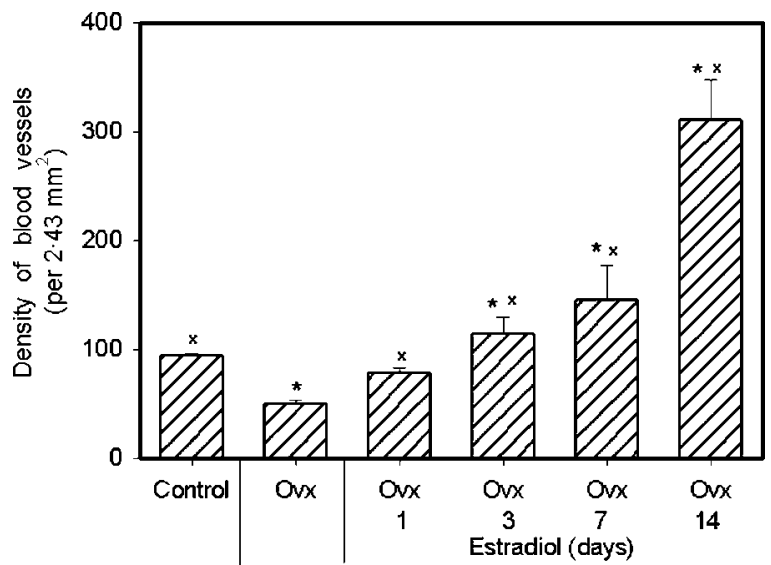

Figure 3 The figure shows the mean density of vessels per group. Blood vessel density significantly decreased after 2 weeks of ovariectomy, which was found to be increased significantly over the 2 week estrogen supplementation period. Each bar is the mean \pm S.E.M. for $N=4$. *Significantly different from control group; $x$, significantly different from Ovx group, $P<0 \cdot 05$.

of ECs: degradation of vascular basement membrane and interstitial matrices by proteases, migration, proliferation, and tube formation. Among them, coordinated reinforcement of matrix degradation and cell migration is characteristic of the angiogenic phenotype of ECs. A number of proteases are required for the degradation of extracellular matrices. Matrix metalloproteinases (MMPs) are indispensable for the degradation of collagen, a major component of extracellular matrices (Davis \& Senger 2005). Estradiol was reported to activate MMP (Klinge et al. 2005). Moreover, estradiol induces endothelial proliferation and migration mediated by the classic estrogen receptor, which is expressed by ECs (Morales et al. 1995, Venkov et al. 1996). VEGF is

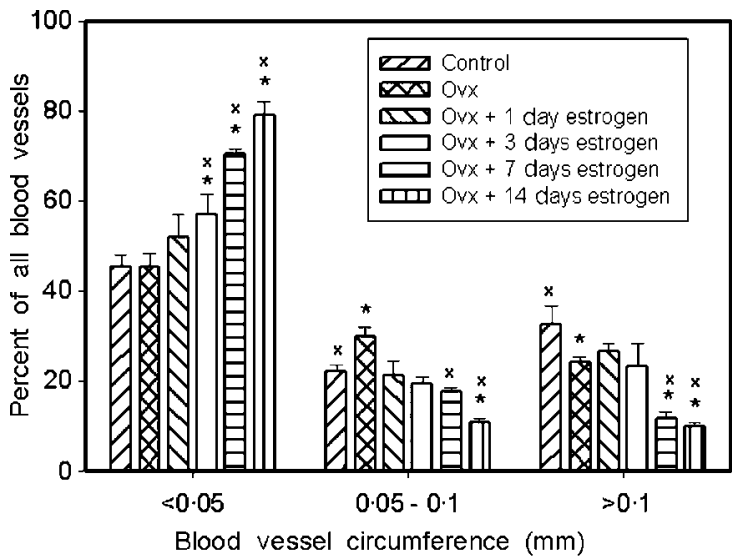

Figure 4 The figure shows the mean circumference of the blood vessels as a percentage of all blood vessels in that group. It is seen that estrogen supplementation mediated a significant shift to smaller size vessels (microvessels). Each bar is the mean \pm S.E.M. for $N=4$. *Significantly different from control group; $x$, significantly different from Ovx group, $P<0 \cdot 05$.

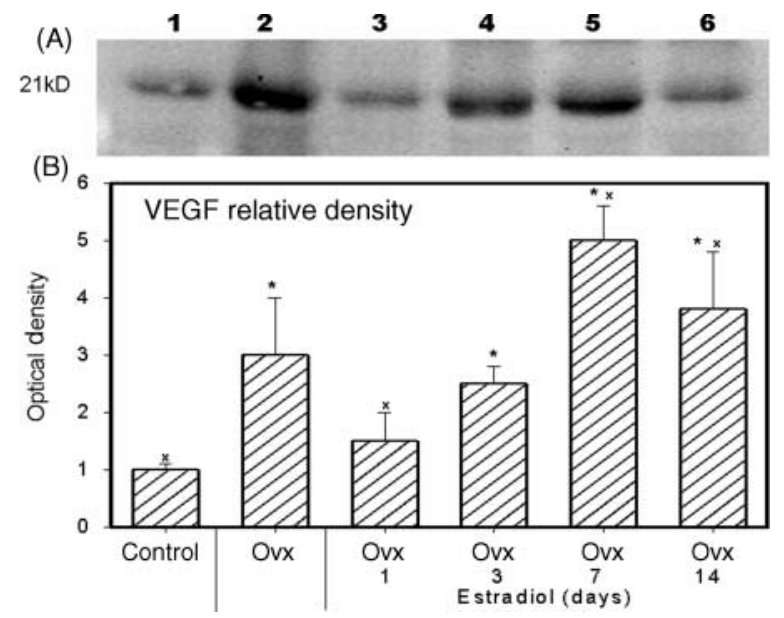

Figure 5 (A) Western blots analyses of VEGF expression in each group. Equal amounts of total extractable proteins $(20 \mu \mathrm{g})$ from control, Ovx and estrogen-treated rabbit bladder smooth muscles were separated by electrophoresis, transferred to membrane and probed with antibody specific to VEGF, as described. Lane 1, control which is a normal female rabbit. Lane 2, 2 weeks after ovariectomy. Lanes 3, 4, 5, and 6 estrogen treated for 1, 3, 7, and 14 days respectively. Note the overexpression of VEGF in Ovx groups, the expression of which is downregulated in 1 and 3 days after estrogen supplementation. By 7 and 14 days, the expression was seen elevated. (B) Average expression of VEGF in different samples. Each bar is the mean \pm S.E.M. for $N=4$. *Significantly different from control group; $x$, significantly different from Ovx group, $P<0.05$.
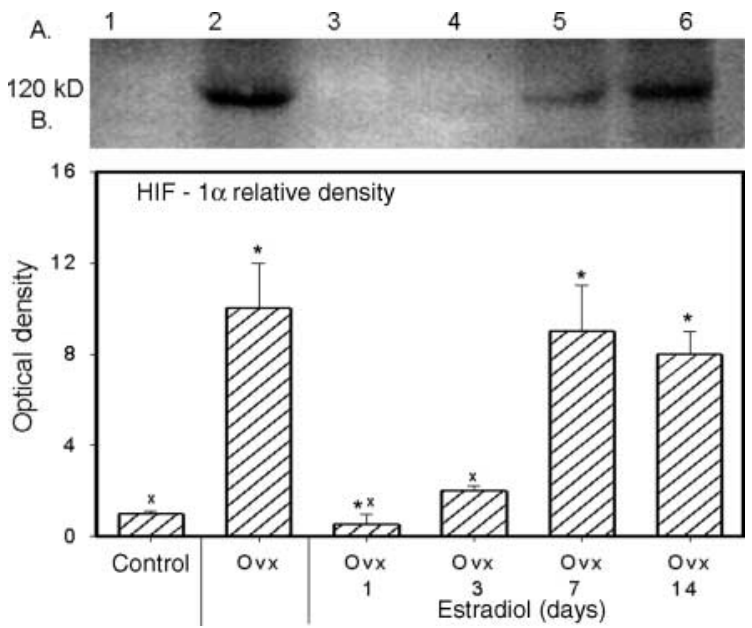

Figure 6 Western blots analyses of HIF- $1 \alpha$ expression in each group. Equal amounts of total extractable proteins $(20 \mu \mathrm{g})$ from control, Ovx and estrogen treated rabbit bladder smooth muscles were separated by electrophoresis, transferred to membrane and probed with antibody specific to HIF- $1 \alpha$, as described. Lane 1, control which is a normal female rabbit. Lane 2, 2 weeks after ovariectomy. Lanes 3, 4, 5, and 6 estrogen treated for 1, 3, 7, and 14 days respectively. Note the fivefold increase in the expression of HIF- $1 \alpha$ in Ovx groups, the expression of which is very low in 1 and 3 days after estrogen supplementation. By 7 and 14 days, the expression was seen elevated. (B) Average expression of HIF-1 $\alpha$ expression in each group. Each bar is the mean \pm S.E.M. for $N=4$. *Significantly different from control group; $x$, significantly different from Ovx group, $P<0.05$. 
thought to play a principal role by stimulating migration and proliferation of ECs and the expression of angiogenesisrelated genes in ECs (Ferrara 2000). VEGF plays a major role in the induction of increased microvascular permeability and in angiogenesis (Neufeld et al. 1999). It may be partially responsible for the angiogenic action of estradiol (Soares et al. 2004). The present results are consistent with the above mechanisms of estrogen-induced angiogenesis.

It is believed that angiogenesis in adults is activated only in response to wound healing and in the females during the reproductive cycle. In pathological circumstances, such as breast cancer, a clear association between estrogens, estrogen receptor expression by ECs, angiogenic activity, and/or tumor invasiveness has been made (Vartanian et al. 1994). Furthermore, onset of neovascularization permits expansion of tumor mass, invasion, and metastasis. Endogenous estrogen levels may also affect the risk of breast cancer. Increased lifetime endogenous estrogen exposure appears to increase breast cancer risk. Those with highest estradiol levels had a twofold increased invasive breast cancer risk, compared with women with lower levels (Lippman et al. 2001). Herein, with the present finding of increased expression of VEGF and estrogen-induced neovascularization, which invaded into smooth muscles extracellular matrices, we might provide an advanced evidence of the estrogen-associated tumor angiogenesis.

Under physiological conditions, angiogenesis is routinely observed in the uterus in association with fluctuations in the levels of circulating estradiol and other sex steroids. This observation suggests the potential for sex steroids to influence neovascularization (Losordo \& Isner 2001) and is consistent with the observations in this study. The phenomenon of neovascularization, which penetrated into the extracellular matrices of the bladder smooth muscle, might indicate the therapeutic stimulation of angiogenesis in conditions such as postmenopausal bladder dysfunction and urinary incontinence, as well as in urogenital wound healing, tissue remolding, and even in in vivo/in vitro tissue engineering in women.

Interestingly, partial outlet obstruction in male rabbits induces a rapid and significant bladder hypertrophy, which is also accompanied by rapid angiogenesis (Chichester et al. 2001) such that the vascular density remains fairly constant during the period of hypertrophy. However, the localization of the neovascularization is between the smooth muscle bundles (following the tracks of the normal vascularization). This results in focal areas of hypoxic smooth muscle within the hypertrophied smooth muscle, which in turn results in both neuronal and smooth muscle damage resulting in contractile dysfunctions. Unlike the angiogenesis observed following partial outlet obstruction, the angiogenesis following estrogen administration results in vascularization within the hypertrophied smooth muscle resulting in no hypoxic areas or neuronal or muscular damage, which in turn results in the 'functional hypertrophy' in which contractile responses are increased in proportion to the level of hypertrophy.

\section{Acknowledgements}

This material is based upon work supported in part by the Office of Research and Development Medical Research Service, Department of Veteran's Affairs and in part by NIH grant RO-1-DK 067114. This work is also supported by the Capital Region Medical Research Foundation. The authors declare that there is no conflict of interest that would prejudice the impartiality of this scientific work.

\section{References}

Aikawa K, Sugino T, Matsumoto S, Chichester P, Whitbeck C \& Levin RM 2003 The effect of ovariectomy and estradiol on rabbit bladder smooth muscle contraction and morphology. Journal of Urology 170 634-637.

Chichester P, Schroder A, Horan P \& Levin RM 2001 Vascular response of the rabbit bladder to chronic partial outlet obstruction. Molecular and Cellular Biochemistry 226 1-8.

Davis GE \& Senger DR 2005 Endothelial extracellular matrix: biosynthesis, remodeling, and functions during vascular morphogenesis and neovessel stabilization. Circulation Research 97 1093-1107.

Ferrara N 2000 Vascular endothelial growth factor and the regulation of angiogenesis. Recent Progress in Hormone Research 55 15-35.

Hu KK, Boyko EJ, Scholes D, Normand E, Chen CL, Grafton J \& Fihn SD 2004 Risk factors for urinary tract infections in postmenopausal women. Archives of Internal Medicine 164 989-993.

Kazi AA, Jones JM \& Koos RD 2005 Chromatin immunoprecipitation analysis of gene expression in the rat uterus in vivo: estrogen-induced recruitment of both estrogen receptor alpha and hypoxia-inducible factor 1 to the vascular endothelial growth factor promoter. Molecular Endocrinology 19 2006-2019.

Klinge CM, Blankenship KA, Risinger KE, Bhatnagar S, Noisin EL, Sumanasekera WK, Zhao L, Brey DM \& Keynton RS 2005 Resveratrol and estradiol rapidly activate MAPK signaling through estrogen receptors alpha and beta in endothelial cells. Journal of Biological Chemistry $\mathbf{2 8 0}$ 7460-7468.

Lippman ME, Krueger KA, Eckert S, Sashegyi A, Walls EL, Jamal S, Cauley JA \& Cummings SR 2001 Indicators of lifetime estrogen exposure: effect on breast cancer incidence and interaction with raloxifene therapy in the multiple outcomes of raloxifene evaluation study participants. Journal of Clinical Oncology 19 3111-3116.

Losordo DW \& Isner JM 2001 Estrogen and angiogenesis: a review Arteriosclerosis, Thrombosis and Vascular Biology 21 6-12.

Morales DE, McGowan KA, Grant DS, Maheshwari S, Bhartiya D, Cid MC, Kleinman HK \& Schnaper HW 1995 Estrogen promotes angiogenic activity in human umbilical vein endothelial cells in vitro and in a murine model. Circulation 91 755-763.

Neufeld G, Cohen T, Gengrinovitch S \& Poltorak Z 1999 Vascular endothelial growth factor (VEGF) and its receptors. FASEB Journal 13 9-22.

Parekh MH, Chichester P, Lobel RW, Aikawa K \& Levin RM 2004 Effects of castration on female rabbit bladder physiology and morphology. Urology 64 1048-1051.

Shweiki D, Itin A, Soffer D \& Keshet E 1992 Vascular endothelial growth factor induced by hypoxia may mediate hypoxia-initiated angiogenesis. Nature 359 843-845.

Soares R, Balogh G, Guo S, Gartner F, Russo J \& Schmitt F 2004 Evidence for the notch signaling pathway on the role of estrogen in angiogenesis. Molecular Endocrinology 9 2333-2343. 
Tinelli A, Tinelli R, Perrone A, Malvasi A, Cicinelli E, Cavaliere V \& Tinelli FG 2005 Urinary incontinence in postmenopausal period: clinical and pharmacological treatments. Minerva Ginecologica 57 593-609.

Vartanian RK \& Weidner N 1994 Correlation of intratumoral endothelial cell proliferation with microvessel density (tumor angiogenesis) and tumor cell proliferation in breast carcinoma. American Journal of Pathology 144 1188-1194.

Venkov CD, Rankin AB \& Vaughan DE 1996 Identification of authentic estrogen receptor in cultured endothelial cells. Circulation 94 727-733.

Vincent KA, Shyu KG, Luo Y, Magner M, Tio RA, Jiang C, Goldberg MA, Akita GY, Gregory RJ \& Isner JM 2000 Angiogenesis is induced in a rabbit model of hindlimb ischemia by naked DNA encoding an HIF-1alpha/ VP16 hybrid transcription factor. Circulation 102 2255-2261.
Weidner N, Semple JP, Welch WR \& Folkman J 1991 Tumor angiogenesis and metastasis: correlation in invasive breast carcinoma. The New England Journal of Medicine 3248.

Received 16 December 2005

Received in final form 16 March 2006

Accepted 5 May 2006

Made available online as an Accepted Preprint 23 May 2006 